Ministry of Education Iraqi Directorate of Education Baghdad Karkh III

First Conference Department of Preparation and Training Division of Research and Studies وزارة التربية العراقية مديرية تربية بغداد الكرخ الثالثة المؤتمر الاول قسم الاعداد والتدريب شعبة البحوث والدراسات

\title{
The Effect of Using Mathematical Representations in the Achievement of Fourth Grade Students in Mathematics
}

\author{
Murooj M. H. Al- Jubouri \\ Directorate of Baghdad Education Karkh III, Ministry of Education, Baghdad, Iraq \\ murooj@rocketmail.com
}

\begin{abstract}
The research sample consists of (71) students distributed over two groups, One experimental (37) students and the other control (34) students, The experimental group has been taught according to use mathematical representations while the control group has been taught according to the traditional method, The two groups have been equalized in the following variables: (previous knowledge test in mathematics, , intelligence test), the researcher has prepared one test is achievement test, The former includes (30) items in (6) questions, Three of them are objective containing (10) true and false items, (9) item of fill in the blank items and (8) multiple - choice of four alternatives and three open - ended questions, Content validity of the test has been secured.
\end{abstract}

Keywords: Fourth grade, mathematics, mathematical representations, formulated zero hypothesis.

\section{إثر استخدام التمثيلات الرياضية في تصصيل تلاميذ الصف الرابع الابتدائي في مادة الرياضيات}

\author{
مروج محمد حمد الجبوري \\ وزارة التربية، مديرية تربية بغداد الكرخ الثالثة، بغداد، العراق \\ murooj@rocketmail.com
}

المستخلص تكونت عينة البحث من (71) تلميذ وزعت إلى مجموعتين إحداهما تجريبية بلغ عدد أفرادها (37) تلميذ والأخرى ضابطة بلغ عدد أفرادها (34) تلميذ ، تم تدريس المجموعة التجريبية على وفق استخدام التمثيلات الرياضية ، بينما تم تدريس المجموعة الضابطة على وفق الطريقة الاعتيادية, وتم مكافأة افراد تلاميذ مجموعتي البحث في المتغيرات الآتية (اختبار المعرفة السابقة في الرياضيات، اختبار الذكاء).أعدت الباحثة اختبار تحصيلي تألف الاختبار من (30) فقرة شملت (6) أسئلة،(3) منها أسئلة

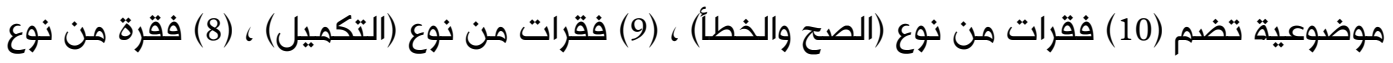

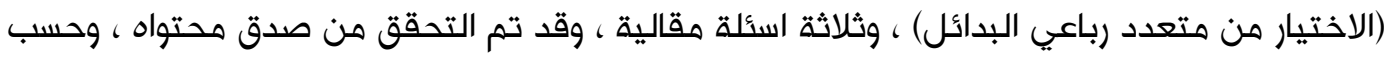

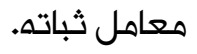

الكلمات الدالة: الصف الرابع، مادة الرياضيات، التمثيلات الرياضية، صيغت الضرضية الصفرية. Al- Jubouri, M. (2019). The Effect of Using Mathematical Representations in the Achievement of Fourth Grade Students in Mathematics. Journal Port Science Research, 2(3), 593-613. doi.org/10.36371/port.2018.02.3.12 
ان مقررات الرياضيات المختلفة دون غيرها من المقررات التعليمية التي يراها البعض هي الاقل تشويقا والاضعف عند المتعلمين , فهم يعانون في فهمها وادراك علاقاتها المجردة , فهي مقرر علمي وتربوي لا يمكن عزله عن المجتمع , ليصبح تعليم وحب الرياضيات ضرورة ملحة , مما يتطلب وجود طرائق تدريسية تلبي متطلبات تدريسها [1], وقليل من المعلمين يتقنون اساليب تقديم الهحاضرات المثيرة للتعليم وادارة النقاش وطرح الاسئة المساعدة , وحل الهشكلات لتنمية تفكير المتعلمين, وحثهم للوصول الى المعرفة واستنتاجها بأنفسهم, واحداث التعلم لديهم بأسلوب يثير دافعيتهم وتعلمهم المستقل [2] وفي ظل الثورة المعلوماتية والتفجير المعرفي السريع والمتلاحق الذي يتميز به عصرنا الحالي اصبح لزاماً على المنظوهة التربوية بكل عناصرها ان تواكب هذا التغيير السريع [3] , لم تعد الاساليب التقليدية في التعليم التي تركز على دور المعلم في الموقف التعليمي وتجعل دور المتعلم سلبياً, يقتصر على مجرد استقبال المعلومات وحفظها واسترجاعها, صالحة في ظل ظروف العصر الحالي الذي يتسم بالتطور العلمي والتقني الهائل في شتى مجالات الحياة , وتطور وسائل الاتصالات والحصول على المعارف [4] , وان لجوء المعلم الى اختيار اسلوب واحد للتعليم وعدم الحيد عنه, يعد ضرباً للجمود الفكري والتربوي الذي يؤدي بالضرورة الى الأساءة لعملية التعلم والتعليم, فالمعلم الناجح هو الذي ينظر في متغيرات الموقف التعليمي ويختار الاسلوب التي يرى انه يتناسب مع ذلك الموقف ومتغيراته [5], وقد تتهم الرياضيات بالكره والنفور من قبل المتعلمين حتى وصفتها دراسة اجريت في بريطانيا بأنها العدو الاول للمتعلمين في مدارسها واشارت الى ارتفاع الرسوب في مادة الرياضيات [6], وان المرحلة الابتدائية من المراحل الههمة إذ أنها تعد الأساس للهراحل اللاحقة فكلما كان الأساس قوياً كان النظام التعليمي أكثر متانة على مواجهة متطلبات العصر لذلك لابد من الاهتمام بتلك المرحلة [7], وقد أثبتت بعض الدرّاسات التربوية العربية والأجنبية أن استخدام أنماط خاصة من التمثيل (مرئية أو ملموسة) تؤدي إلى تحسين قدرات التلاهيذ الرياضية وههارات التفكير وحل المشكلات , منها دراسة ( إبراهيم, 2001) [8] التي هدفت إلى الكشف عن مقدرة طلبة الصفين السابع والثامن على التمثيل الجبري والهندسي للمسألة الرياضية اللفظية وأسفرت نتائج التحليلات الإحصائية عن وجود تدني في مستوى مقدرة طلبة الصفين السابع والثامن على التمثيل الجبري والهندسي للمسالة الرياضية الجبرية , وعلى الرغم من أن دراسات كثيرة تناولت اثر طرائق واستراتيجيات متعددة في تحصيل التلاميذ في الرياضيات فان استخدام التمثيلات الرياضية لم تحظ بالقدر الكافي من الاهتمام وقد يعود ذلك إلى حداثة المعيار , وترى الباحثة أن واقع تعليم

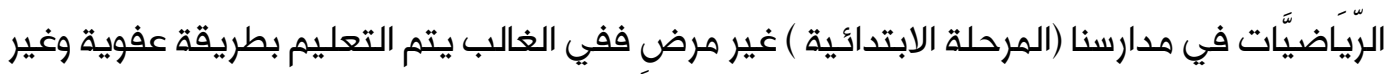
مخطط لها مسبقاً من المعلم. وتكمن مشكلة البحث الحالي في أن مؤسستنا التربوية والتعليمية مازالت تعتمد على الحفظ والتلقين في تعليم الرياضيات وان عدم استخدام طرائق تدريس الجبوري، مروخ محمد حمد. (2019). إثر استخدام التمثيلات الرياضية في تحصيل تلاميذ الصف الرابع الابتدائي في مادة doi.org/10.36371/port.2018.02.3.12 الرياضيات. Journal Port Science Research ، 
واستراتيجيات حديثة فعالة أدى إلى تدني المستوى العلمي للتلاميذ ومن ثم تدني التحصيل الدراسي وهذا ما أسفرت عنه نتائج أكثر الدراسات العراقية التي أجريت في هذا الهيدان، إذ أشارت اغلبها إلى تدني التحصيل الدراسي للتلاميذ في مادة الرياضيات.

\section{•}

لقد تطورت طرائق التعليم شيئا فشيئا شأنها في ذلك شأن اية ظاهرة في الحياة تبدأ بسيطة سطحية, ولكنها بعد زمن يطول او يقصر تضرب بجذورها في الارض , وتأخذ مدياتها العلمية التي تعزز من وجودها فتصبح آنذاك ظاهرة يستشهد بها العلماء والباحثون وطلبة العلم, وفي ظل ما تنادي به كل السياسات التربوية بتحقيق المواطنة والانتماء والعدل , نجد انفسنا مطالبين بتوفير كل ما من شأنه مساعدة الهتعلمين على تحفيز الدافعية الذاتية نحو التعلم في حماس وديناميكية لمسايرة حركة الحياة بإنتاج وفاعلية نحو تحقيق هذه الاهداف [9]، ان تقدم الامم والشعوب في الوقت الحاضر لا يعتمد فقط على ما لديهم من إمكانات مادية , ولكن يعتمد ايضاً على ما لديهم من إمكانات بشرية تتمثل بالأفراد المبتكرين المبدعين الذين لديهم القدرة على مواجهة المشكلات والعمل على حلها في ميادين الحياة كافة [10], وإن خبراء التعليم في جميع أنحاء العالم يتجهون نحو نهط من التعليم يركز في جوهره على تعليم المتعلمين التفكير بأنماطه المختلفة , مع اتخاذ اسلوب حل المشكلات كأحد أبرز اساليب التعلم التي سادت المناهج التعليمية , وما زالت تسودها حتى وقتنا الحاضر [11] , وان التفكير سلوك راقٍ, ويُعد من ارقى انواع السلوك البشري, فهو يساعدنا على التخطيط الجيد وعلى محاكاة الاشياء والوصول الى القرار الحكيم, وبالتفكير نبتكر ونخترع

$$
\text { ونكتشف ونحل المشكلات [12]. }
$$

ويعدُ التمثيل أداة مهمة للتفكير حيث أنه يجعل الأفكار الرياضية أكثر حسية وينمي الاستدلال من خلال مساعدة التلاميذ في التركيز على مظاهر مهمة من الموقف الرياضي، كذلك فهو يساعد التلاميذ على أدراك العناصر الرياضية المشتركة بين المواقف المختلفة [13] (ويؤكد .Hwang\& et) (al. , 2007) الإبداعي لديهم ، وأن المعلمين بحاجة إلى تقييم التلاميذ في إجراءات حل المشكلات من خلال تمثيلها سواء أكان بصيغ أو رسوم بيانية، وبذلك يستطيع المعلمون التأكد من أن التلاميذ يسيئون فهم مفهوم معين أو عالقون في نقطة محددة [14] , وقد أجريت الكثير من الدراسات الأجنبية التي استخدمت التمثيلات الرياضية , وأشارت تلك الدراسات إلى أهمية استخدام التمثيلات في تعليم مادة الرياضيات لما لها من اثر ايجابي في تعلم التلاميذ, ويمكن تلخيص أهمية هذا البحث من خلال أهمية التمثيلات الرياضية في تحصيل التلاميذ الصف الرابع الابتدائي.

Al- Jubouri, M. (2019). The Effect of Using Mathematical Representations in the Achievement of Fourth Grade Students in Mathematics. Journal Port Science Research, 2(3), 593-613. doi.org/10.36371/port.2018.02.3.12 
يهدف البحث الحالي إلى: التعرف على أثر التمثيلات الرياضية في تحصيل تلاميذ الصف الرابع الابتدائي في مادة الرياضيات.

\section{فرضيــة البحث}

لا يوجد فرق ذو دلالة إحصائية عند مستوى الدلالة (0,05) بين متوسط درجات تلاهيذ (المجموعة التجريبية) الذين يدرسون وفق استخدام التمثيلات الرياضية ومتوسط درجات تلاميذ (المجموعة الضابطة) الذين يدرسون وفق الطريقة الاعتيادية في الاختبار التحصيلي.

يقتصر البحث الحالي على: تلاميذ الصف الرابع الابتدائي في المدارس الابتدائية في المديرية العامة لتربية بغداد/الكرخ الثالثة، مدرسة الشهيد عبد اللطيف علوان الابتدائية المختلطة، الفصل الدراسي الأول للعام 2018 - 2019 م، كتاب الرياضيات المقرر للصف الرابع الابتدائي ط 1 لسنة 2018 م.

$$
\begin{aligned}
& \text { • تحديد الهصطلحات: } \\
& \text { التمثيل الرياضي: عرفه كل من: }
\end{aligned}
$$

( بهوت وعبد القادر,2005) بأنه ترجمة النص الرياضي من أحد أشكاله (ألفاظ أو كلمات، جداول، رموز، أشكال، علاقات رياضية) إلى نماذج محسوسة أو شكل آخر من أشكاله [15]. (السواعي، 2010) وهو استخدام أشياء مثل الكلمات والرموز والرسومات والجداول والمواد المحسوسة ... الخ للتعبير عن فكرة أو مفهوم رياضي [13]. تبنت الباحثة تعريف (بدوي، 2003) بأنه " ترجمة المسألة أو الفكرة الرياضية إلى صيغة جديدة أو شكل توضيحي أو جدول للمعلومات أو شكل بياني أو نموذج حسي أو ترجمة المسائل المصورة إلى رموز وكلمات رياضية " [16]. التهريف الاجرائي للتمثيلات الرياضية: هو استخدام صور للتعبير اللفظي كالرموز، او التعبير غير اللفظي من رسوم واشكال بيانية ومخططات وجداول وكذلك استخدام مواد محسوسة للتعبير عن عناصر المحتوى الرياضي. التحصيل: عرفه كل من: (Alderman,2007) بأنه "إثبات القدرة على أنجاز ما تم اكتسابه من الخبرات التعليمية التي وضع

$$
\text { من أجله"[17]. }
$$

الجبوري، مروخ محمد حمد. (2019). إثر استخدام التمثيلات الرياضية في تحصيل تلاميذ الصف الرابع الابتدائي في مادة

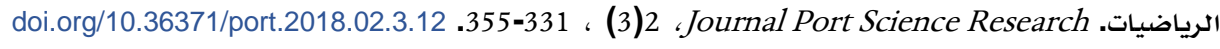


(نصر الله,2010): بأنه العلامة (الرقم القياسي التحصيلي) الذي استطاع ان يحصل عليها المتعلم في

الاهتحان التحصيلي المدرسي، واعتمد او سجل او رصد من قبل المعلم خلال مدة زمنية معينة [18].

التعريف الإجرائي للتحصيل:

هي الدرجة التي يحصل عليها تلاميذ عينة البحث الحالي عند إجابتهم عن فقرات الاختبار التحصيلي الذي أعد لهذا الغرض. لهن.

\section{الخلفية النظرية والدراسات السابقة}

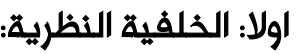

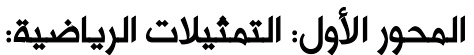

بالرغم من حدوث تغييرات في مناهج وكتب الرياضيات في العقود الاخيرة، الا ان التطويرات والتغييرات التي طرأت على اساليب التعليم وطرائقها ما تزال دون التطور والتغيير الذي طرأ على المناهج والكتب، ويمكن ان يعزى ذلك الى ضعف اهتمام واضعي الكتب المدرسية بنتائج الابحاث والدراسات التي تجري في هذا المجال والتي أثبتت تفوق الطرائق الحديثة على غيرها من طرائق التعليم المعتمدة، وبرز معيار التمثيل الرياضي كأحد معايير العمليات في وثيقة " المبادئ والمعايير " الصادرة عام 2000 كمعيار مستقل، بعد أن كان وارداً بشكل ضمني كأحد مؤشرات معيار التواصل في وثيقة عام 1989، ويرى (Fennel\& Rowan, 2001) أنه تم إعطاء التمثيل أهمية كبرى عند تحديث المبادئ والمعايير عام 2000 نظراً لأهمية الدور الذي يلعبه لكونه أداة للاتصال والتفكير [19]، ويعدُ (Pape \& Tchosnov, 2001 ) التمثيل بأنه عملية يتم من خلالها التفاعل بين مدخلات التمثيل الخارجي مع الصور الذهنية، ويجري تعلم المفاهيم الرياضية من خلال بناء تدريجي للصور الذهنية للمفاهيم الأولية، كما يعتقد وجود تأثير متبادل بين شكل التمثيل الداخلي والخارجي، وأن طبيعة كل منهما يؤثر في الآخر, ويوضح ( Pape \& Tchosnov ) ذلك التفاعل بالرسم الآتي:

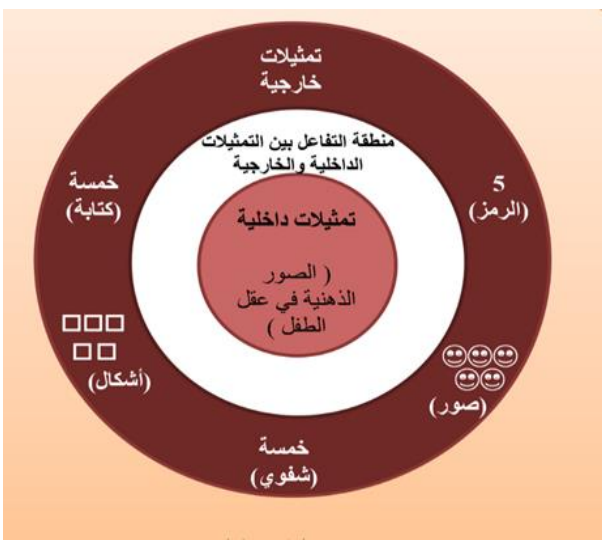

الشكل (1) يمثل العلاقة بين التمثيلات الداخلية في عقل الطفل والتمثيلات الخارجية

Al- Jubouri, M. (2019). The Effect of Using Mathematical Representations in the Achievement of Fourth Grade Students in Mathematics. Journal Port Science Research, 2(3), 593-613. doi.org/10.36371/port.2018.02.3.12 
يلاحظ في الشكل السابق العلاقة بين التمثيلات الداخلية في عقل الطفل والتمثيلات الخارجية التي يمكن إدراكها من خلال الكلام أو الكلمات أو الرموز أو الصور أو الأشكال، كما يوضح وجود منطقة في المنتصف بين التمثيلات الداخلية والخارجية هي منطقة التفاعل التي يتم من خلالها التعبير عن الصور الذهنية خارجياً، أو استيعاب التمثيلات الخارجية على شكل صور ذهنية [20], ويقسم ( Golden ( ) Shteingold للفكرة الواحدة التي تقدم للتلميذ مثل الصور، الصيغ/ الرسوم الإحصائية، الرموز، والمحسوسات، واللغة المحكية, والقسم الثاني هو تلك التمثيلات الداخلية أي الصور الذهنية التي يبنيها التلميذ للمفهوم [21], كما يؤكد (Salkind , 2007) أهمية استخدام التمثيلات الرياضية في تدريس الرياضيات في المدرسة، وأهمية التمثيلات سواء أكانت ملموسة، أو تصويرية، أو رمزية، أو لفظية أو بصرية، أو داخلية أو خارجية في تنمية التواصل وحل المشكلات. كما أشارت إلى ضرورة استخدام المعلمين للتمثيلات بشكل فعال أثناء تدريس المحتوى لتحقيق المعرفة العميقة في الرياضيات [22]، " كما يساعد تعلم الأشكال المختلفة للتمثيل التلاميذ في فهم المفاهيم والعلاقات الرياضية، وتبليغ تفكيرهم وحججهم وفهمهم، وتعرَّف الترابطات بين الهفاهيم الرياضية ذات العلاقة، واستخدام الرياضيات لنمذجة وتفسير مواقف المشكلات الواقعية، وتعرَّف الارتباطات بين التمثيلات، واستخدام التمثيلات المختلفة بشكل ملائم وحسب الحاجة لحل المشكلات " [23] ويرى ( Mousley , 2004 ) أن الاستخدام المتزايد لتمثيل الهفاهيم الرياضية من شأنه تطوير مسارات التعلم لدى التلاميذ ، التي تمكن التلاميذ من بناء روابط معرفية بين الهفاهيم [24] ويعتبر التمثيل أداة مهمة للتفكير حيث أنه يجعل الأفكار الرياضية أكثر حسية وينمي الاستدلال من خلال مساعدة التلاميذ في التركيز على مظاهر مهمة من الموقف الرياضي , كذلك فهو يساعد التلاهيذ على ادراك العناصر الرياضية المشتركة بين المواقف المختلفة، حيث يشير كل من (Suh \& Moyer, 2007) إلى أن مجموعة متنوعة من التمثيلات تزيد من فرص المتعلمين لفهم الأفكار الرياضية ، لأنه عندما يقوم المتعلمين بترجمة وإعادة تفسير الأفكار من تمثيل لآخر تجعل الاتصال المفاهيمي أكثر عمقـاً لـدى

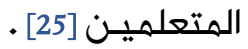

أهمية التمثيل الرياضي: تأتي أهمية التمثيل الرياضي من ارتباطه بكافة مجالات الرياضيات،

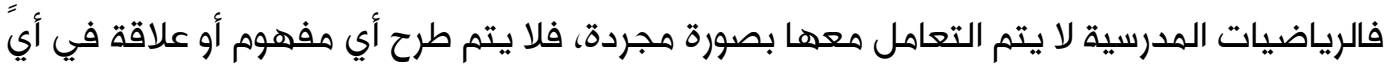
من مجالاتها إلا ويكون مرتبطاً بتمثيل أو عدة تمثيلات توضح هذا المفهوم، سواء أكان ذلك في الحساب، الجبر، الهندسة، القياس، والاحتمالات فجميع المفاهيم ترتبط بتمثيل يجسدها ويقربها من ذهن المتعلم ، ويذكر ( Pape\&Tchoshanov,2001 ) ان تعلم المفاهيم يأتي عبر البناء التدريجي

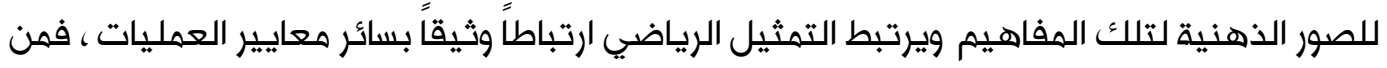


جهة يعمل التمثيل على إيصال المفاهيم والعلاقات المجردة بصور ورموز وإشكال متعددة، ومن جهة أخرى يسهل التواصل بين المتعلمين بعضهم ببعض و بين المتعلمين والمعلم حول مفاهيم ذات رموز محددة ومتفق عليها [26] نستخلص من خلال عرض الأفكار السابقة أهمية استخدام التمثيلات الرياضية في فهم الأفكار الرياضية, وان استخدام التمثيلات الرياضية مهمة لتوضيح فكرة رياضية أو موقف رياضي أو مفهوم رياضي, فاستخدام التمثيلات الرياضية يسهح للتلاميذ بالتعامل مع الأشكال المختلفة للمفاهيم الرياضية والأفكار الرياضية , مما يسهل عليهم استيعاب الأفكار والمفاهيم والمواقف الرياضية , فضلاً عن ما سبق فإن (Sun \& others,2009) يشيران إلى عدة دراسات أجمعت على أن استخدام التمثيلات للمفاهيم والأفكار الرياضية وترابط تلك المفاهيم بطرق ذات معنى للمتعلمين يعد محكاً لتحديد ما إذا كانت الكتب الدراسية أو معلمو الرياضيات يعملون على بناء المفاهيم والأفكار الرياضية بشكل عميق لدى المتعلمين [27].

المحور الثاني التحصيل: يولى المعنيون بالتعليم التحصيل الدراسي اهتماماً كبيراً نظراً لأهميته في حياة الفرد وما يترتب على نتائجه من قرارات تربوية حاسهة، فالاختبارات التحصيلية وسيلة منظمة تهدف إلى قياس كمية المعلومات التي يحفظها المتعلم أو يتذكرها في حقل من حقول المعرفة، كما تشير الى قدرته على فهمها أو تطبيقها، وتحليلها والانتفاع بها في مواقف الحياة المختلفة [28].

\section{العوامل المؤثرة في التحصيل:}

هناك عدد من العوامل المؤثرة في عملية التحصيل ومنها: 1) مقدار ما يتمتع به المتعلم من ذكاء عام وقدرات خاصة وميول واستعدادات ومهارات وخبرات

$$
\text { وغيرها. }
$$

2) مقدار ما يوجد لدى المتعلمين من دوافع وشعور بالحماسة والاهتمام بالدراسة وبذل الجهد والطاقة. 3) مقدار ما يتمتع به المتعلم من السلامة الجسمية والصحة العقلية والنفسية. 4) طرائق التدريس وما يرافقها من إثارة وتشويق وجذب لانتباه المتعلمين، واستعمال الوسائل والتقنيات التعليمية المناسبة، وإشراك المتعلم في النشاط التعليمي وغيرها. 5) شخصية المعلم ومقدار ما يتمتع به من القدرة على نقل المعلومات وهدى تمكنه من المادة العلمية. 6) مقدار ما يتوافر من الكتب والمصادر الجيدة والحديثة فضلاً عن توافر الورش والمختبرات. 7) مقدار تفرغه للدراسة، وعدم تكليفه بالقيام بأعباء والتزامات خارجية.

$$
\text { 8) ظروف السكن والإقامة التي يعيش فيها. }
$$

9) مقدار ما يتمتع به من الجو العائلي الهادئ والملائم للدراسة [29].

Al- Jubouri, M. (2019). The Effect of Using Mathematical Representations in the Achievement of Fourth Grade Students in Mathematics. Journal Port Science Research, 2(3), 593-613. doi.org/10.36371/port.2018.02.3.12 
في ضوء مشكلة البحث وأهدافها، نتناول عدداً من الدراسات السابقة والتي تتصل بمشكلة البحث الحالي وهي كما موضح ادناه.

دراسة السعيدي في الأردن 2010 "فاعلية استخدام التمثيلات الرياضية المتعددة في تنمية مهارات حل المشكلات الرياضية لدى طلاب الصف الثامن من التعليم الأساسي" المستوى العلمي لعينة الدراسة الصف الثامن الأساسي، نوع التصميم وعدد وحجم العينة التصميم التجريبي تكونت عينة الدراسة من مجموعتين تجريبية وتكونت من 66 طالب وطالبة وضابطة تكونت من 62 طالب وطالبة، أدوات الدراسة اختبار مهارات حل المشكلات الرياضية، النتائج فاعلية التمثيلات الرياضية في تنمية مهارات حل المشكــلات الرياضيـة لـدى طـلاب الصـف الثامـن الأساسي [30]. دراسة Grossman, 2010 في أمريكا "إثر استخدام التمثيلات الرياضية المتعددة من اجل بناء تعاون أقوى بين الطلاب وتنمية تفكيرهم في حل المسائل الرياضية" المستوى العلمي لعينة الدراسة الصف الثامن للمرحلة الإعدادية، نوع التصميم وعدد وحجم العينة التصميم التجريبي تكونت العينة من 16 طالب وتم تقسيمهم إلى مجموعتين تجريبية تكونت من 8 طلاب وضابطة تكونت من 8 طلاب، أدوات الدراسة اختبار تحصيلي بعدي، النتائج وجود فروق ذات دلالة إحصائية للمجموعة التجريبية الذي طبق عليهم التمثيلات المتعددة، وأظهرت الدراسة ميل الطلاب إلى استخدام التمثيلات الأيسر إلى عقولهم [31].

دراسة Kuchemann \& et.,2011, في إنجلترا "أثر استخدام التمثيلات الرياضية والنماذج في تعلم الجبر والمنطق المضاعف وتوضيح المعنى باستخدام خطوط الاعداد المزدوجة في إنجلترا" المستوى العلمي لعينة الدراسة الصف الثامن الثانوي، نوع التصميم وعدد وحجم العينة التصميم التجريبي تكونت العينة من 15 مدرسة وتم اختيار الطلاب التي أعمارهم بين سن (12-13) سنة، أدوات الدراسة تم جمع البيانات من خلال اختبارات نسبة الهضاعف والهنطق واختبار في الجبر، النتائج فعالية البرنامج المستخدم في حل بعض المشكلات وأثر البرنامج في زيادة ثقة الطلاب في التعامل مع المفاهيم الجبرية [32].

\section{2. منهج البحث واجراءاته}

\section{أولاً: التصميم التجريبي:}

اختارت الباحثة التصميم التجريبي ذا الضبط الجزئي لمجموعتين متكافئتين ذات الاختبار البعدي لأنه مناسباً أكثر لتحقيق أهداف البحث، والجدول (1) في أدناه يوضح التصميم التجريبي لعينة البحث 


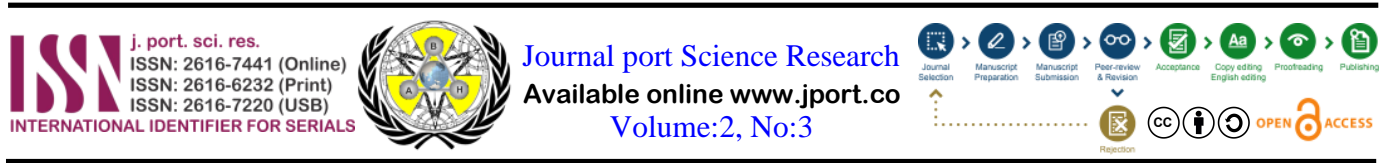

\begin{tabular}{|c|c|c|c|c|c|}
\hline \multicolumn{6}{|c|}{ جدول (1) التصميم التجريبي لعينة البحث } \\
\hline الاختبار البعدي & المتغير التابع & المتغير المستقل & \multirow{3}{*}{ 司 } & المجموعة & ت \\
\hline \multirow[t]{2}{*}{ 1) اختبار التحصيل } & \multirow[t]{2}{*}{ التحصيل } & استخدام التمثيلات & & المجموعة التجريبية & 1 \\
\hline & & الطريقة الاعتيادية & & المجموعة الضابطة & 2 \\
\hline
\end{tabular}

\section{ثانياً: مجتمع البحث:}

يتألف مجتمع البحث الحالي من تلاميذ الصف الرابع الابتدائي في المدارس الابتدائية التابعة إلى

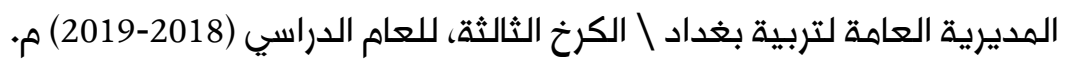

\section{ثالثاً: عينة البحث:}

تم اختيار مدرسة الشهيد عبد اللطيف علوان الابتدائية المختلطة التابعة لمديرية تربية الكرخ الثالثة,

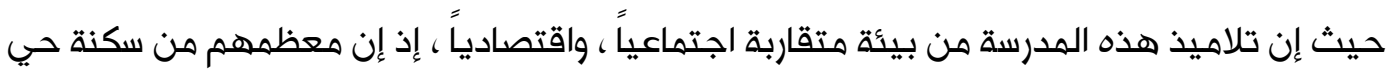
واحد وهذا ما يسهل للباحثة تثبيت بعض المتغيرات بين المجموعتين(التجريبية و الضابطة ) لغرض التكافؤ, وبالاختيار العشوائي تم تحديد شعبتين من أصل ثلاثة شعب للصف الرابع الابتدائي لتمثل إحداهما المجموعة التجريبية وهي شعبة (أ) والأخرى الهجموعة الضابطة وهي شعبة (ب)، وكان عدد تلاميذ المجموعة التجريبية (37) تلميذ ، وعدد تلاميذ المجموعة الضابطة (34) طالبة، بعد استبعاد التلاميذ الراسبين إحصائيا والبالغ عددهن (5) طالبات من المجموعتين التجريبية والضابطة , كي لا تؤثر خبرتهم السابقة في نتائج البحث , وكان عدد أفراد العينة النهائي (71) .

رابعا: اجراءات الضبط:

\section{• السلامة الداخلية للتصميم التجريبي:}

من اجل التأكد من السلامة الداخلية للتصميم التجريبي وتحقيق التكافؤبين تلاميذ مجموعتي البحث قبل تطبيق التجربة، أجرت الباحثة التكافؤ في بعض المتغيرات التي قد تؤثر في نتائج البحث، وهي (اختبار المعرفة السابقة في الرياضيات، اختبار الذكاء).

اختبار المعرفة السابقة في الرياضيات: عدت الباحثة اختبار المعرفة السابقة في الموضوعات التي لها صلة بمادة البحث الحالي من نوع الاختيار من متعدد وبالاستعانة بمعلمات الرياضيات وقد بلغ عدد فقرات الاختبار (20) فقرة موضوعية من نوع الاختيار من متعدد بواقع اربعة بدائل لكل فقرة تم

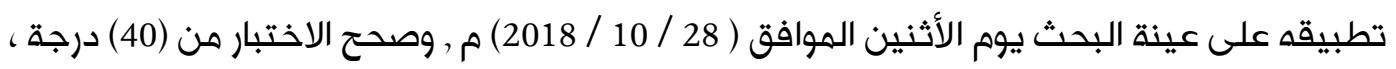
أخذت درجات مجموعتي البحث، وبحساب متوسط الدرجات والتباين لكل مجموعة وباستخدام الاختبار التائي (t-Test) لعينتين مستقلتين غير متساويتين , عند مستوى دلالة (0,05) ظهر أن القيمة التائية

Al- Jubouri, M. (2019). The Effect of Using Mathematical Representations in the Achievement of Fourth Grade Students in Mathematics. Journal Port Science Research, 2(3), 593-613. doi.org/10.36371/port.2018.02.3.12 
المحسوبة اقل من القيمة التائية الجدولية، وهذا يدل على أن المجموعتين متكافئتان في متغير المعرفة السابقة كما موضح في الجدول (2).

جدول (2) نتائج الاختبار التائي في متغير (المعرفة السابقة)

\begin{tabular}{|c|c|c|c|c|c|c|c|c|}
\hline مسإحصائية عند الدلالة & التائية الجيهة & التائية الميهة & الحرجة & التباين & الانحراف & الحتوسط & العدد & المجموعة \\
\hline \multirow{2}{*}{ غير دال } & \multirow{2}{*}{2,000} & \multirow{2}{*}{0,712} & \multirow{2}{*}{69} & 4,157 & 2,039 & 6,810 & 37 & تجريبية \\
\hline & & & & 5,400 & 2,324 & 6,441 & 34 & ضابطة \\
\hline
\end{tabular}

اختبار الذكاء: لغرض إجراء التكافؤ في متغير الذكاء اعتمدت الباحثة اختبار (دانيلز) للاستدلال

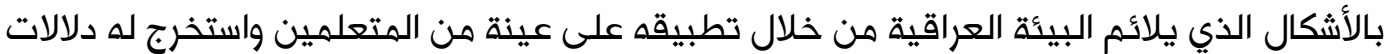
الصدق والثبات. تصبح الدرجة النهائية (45) درجة لـ اختبار الذكاء ، اتبعت الباحثة تعليمات تطبيق الاختبار بدقة على طالبات المجموعتين التجريبية والضابطة لدى تطبيقه يوم الاحد الموافق (2018/10/29) م ، بعد تصحيح الإجابات بإعطاء درجة واحدة للإجابة الصحيحة وصفر للإجابة الخاطئة , ملحق (6) ، وعند إجراء المقارنة بين متوسطي الهجموعتين باستخدام الاختبار التائي (t-Test) لعينتين مستقلتين غير متساويتين لمعرفة دلالة الفرق بين متوسطي ذكاء طالبات مجموعتي البحث ظهر أن القيمة التائية المحسوبة أقل من القيمة التائية الجدولية عند مستوى دلالة (0,05) ودرجة حرية (69) ، وهذا يدل على أن المجموعتين متكافئتان في متغير الذكاء لان الفرق بين المتوسطين غير دال إحصائياً كما موضح في الجدول (3) .

جدول (3) نتائج الاختبار التائي في متغير (الذكاء)

\begin{tabular}{|c|c|c|c|c|c|c|c|c|}
\hline ラ 寻 手 & 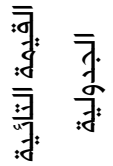 & 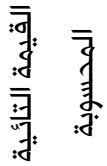 & $\frac{\frac{1}{7}}{\frac{1}{7}}$ & 震 & $\frac{\bar{y}}{\bar{g}} \frac{\bar{g}}{\bar{y}}$ & 亲音 & $\begin{array}{l}y \\
7 \\
\overline{7} \\
:=\end{array}$ & ఫू. \\
\hline \multirow{2}{*}{ غير دال إحصائياً } & \multirow{2}{*}{2,000} & \multirow{2}{*}{0,650} & \multirow{2}{*}{69} & 15,586 & 3,948 & 13,459 & 37 & تجريبية \\
\hline & & & & 18,507 & 4,302 & 12,823 & 34 & ضابطة \\
\hline
\end{tabular}

الجبوري، مروخ محمد حمد. (2019). إثر استخدام التمثيلات الرياضية في تحصيل تلاميذ الصف الرابع الابتدائي في مادة doi.org/10.36371/port.2018.02.3.12 الرياضيات. Journal Port Science Research ، 
تحديد المادة التعليمية (المحتوى) لتجربة البحث: تم تحديد المادة التعليمية التي تُعلم في أثناء التجربة، من كتاب الرياضيات المقرر تعليمه للصف الرابع الابتدائي، والمتضهنة ثلاثة فصول هي: الفصل الرابع: الاحصاء والاحتمالات الفصل الخامس: الضرب والفصل السادس: القسمة [33]. صياغة الأهداف السلوكية : تم صياغة الأهداف السلوكية في صورة نتائج تعليمية نهائية محددة وواضحة وقد اشتقت هذه الأهداف من محتوى المادة التعليمية المقررة للفصول الثلاثة (الرابع و الخامس و السادس) من كتاب الرياضيات للصف الرابع الابتدائي، وقد بلغ عدد الأهداف السلوكية التي صيغت (100) هدفاً سلوكياً, وقد عرضت هذه الأهداف على عدد من المحكمين والمتخصصين ، لبيان آرائهم في مدى وضوحها ودقة صياغتها وتحديد المستوى الذي تقيسه كل فقرة ، وقد اعتمدت نسبة الاتفاق بينهم بـ (80\%) فما فوق ، وقد تم إجراء التعديلات عليها في ضوء آرائهم ومقترحاتهم وملاحظاتهم.

إعداد الخطط التدريسية اليومية : أعدت الباحثة الخطط التدريسية للموضوعات التي درستها لتلاميذ المجموعة التجريبية التي درست على وفق استخدام التمثيلات الرياضية والمجموعة الضابطة التي درست على وفق الطريقة الاعتيادية كلاً حسب مجموعته، بلغ عددها (37) خطة لكل مجموعة وقد عرضت الباحثة خطتين أنموذجيتين على مجموعة من الخبراء وبعض المتخصصين في طرائق التدريس وهعلمي مادة الرياضيات في المدرسة التي طبق فيها الاختبار، للإفادة من آرائهم وتوجيهاتهم وقد اعتمدت نسبة الاتفاق بينهم بـ (85\%) فما فوق، وفي ضوء هذه النسبة اجري التعديل في تلك الخطط من أجل الوصول إلى صورتها النهائية. أداة البحث: قد تطلب البحث الحالي أداة لقياس المتغير التابع وهو: الاختبار التحصيلي. الاختبار التحصيلي: من أجل تحقيق أهداف البحث الحالي تم اعداد الاختبار التحصيلي الهناسب

$$
\text { لأغراض البحث على وفق الخطوات الآتية: }
$$

• صياغة الأهداف السلوكية: لتحقيق هذه الخطوة صِيغ (100) هدف سلوكي موزعة بين المستويات (التذكر، الاستيعاب، والتطبيق) من مســـتويات المجال المعــرفي لبلـوم (Bloom).

إعداد جدول المواصفات: أعدّت الباحثة جدول المواصفات لمحتوى المادة قيد البحث، وبحسب المبر

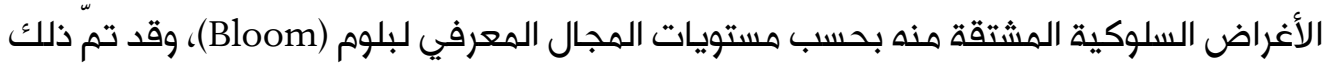
على وفق.

Al- Jubouri, M. (2019). The Effect of Using Mathematical Representations in the Achievement of Fourth Grade Students in Mathematics. Journal Port Science Research, 2(3), 593-613. doi.org/10.36371/port.2018.02.3.12 
جدول (4) جدول المواصفات الخاص بالاختبار التحصيلي (الخارطة الاختبارية)

\begin{tabular}{|c|c|c|c|c|c|c|c|c|}
\hline \multirow{2}{*}{ 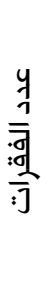 } & \multicolumn{3}{|c|}{ المستويات المعرفية } & \multirow{2}{*}{ 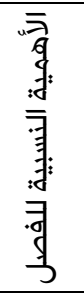 } & \multirow{2}{*}{ 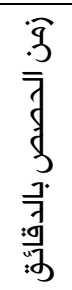 } & \multirow{2}{*}{$\begin{array}{l}y \\
7 \\
\bar{y} \\
\frac{7}{3}\end{array}$} & \multirow[b]{2}{*}{ التعليمي المحتوى } & \multirow[b]{2}{*}{$ت$} \\
\hline & التطبيق 30٪ & الاستيعاب & التذكر & & & & & \\
\hline 9 & 3 & 4 & 2 & $\% 24$ & 405 & 9 & الفصل الرابع & 1 \\
\hline 12 & 4 & 5 & 3 & $\% 41$ & 675 & 15 & الفصل الخامس & 2 \\
\hline 9 & 3 & 4 & 2 & $\% 35$ & 585 & 13 & الفصل السادس & 3 \\
\hline 30 & 10 & 13 & 7 & $\% 100$ & 1665 & 37 & المجموع & \\
\hline
\end{tabular}

إعداد فقرات الاختبار: أعدت الباحثة اختباراً تحصيلياً، مكوناً من (30) فقرة ضمت (6) ستة أسئلة، (3) ثلاثة منها أسئلة موضوعية، اذ ان الأسئلة الموضوعية تمتاز بالموضوعية والمرونة وذات صدق وثبات عاليين، ضم السؤال الأول (10) فقرات من نوع (الصح أو الخطأ)، والسؤال الثاني (9) فقرات من نوع (التكميل)، والسؤال الثالث (8) فقرة من نوع (الاختيار من متعدد) و(3) ثلاثة من الأسئلة المقالية، وقد توزعت فقرات الاختبار التحصيلي على مستويات بلوم (Bloom) الثلاثة (معرفة،

$$
\text { استيعاب، تطبيق) من المجال المعرفي. }
$$

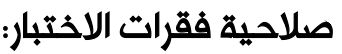

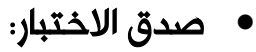

أ) الصدق الظاهري: قد تحقق الصدق الظاهري من خلال عرض فقراته على مجموعة من الخبراء ومجموعة من المختصين في الرياضيات وطرائق تدريسها وأشاروا إلى بعض التعديلات اللازمة، وفي ضوء آرائهم تم حذف بعض الفقرات والإبقاء على الفقرات الاخرى واجراء التعديلات عليها وقد اعتمدت الباحثة نسبة اتفاق 91\% فما فوق. ب) صدق المحتوى: من الأمور التي اعتمدتها الباحثة في صدق الاختبار هو إعداد جدول المواصفات، لمعرفة مدى تمثيل الفقرات لمحتوى المادة التعليمية والأهداف السلوكية، وأن استخدام جدول المواصفات يُعد مؤشراً من مؤشرات صدق المحتوى للاختبار، إذ يمثل درجة مقبولة من صدق تمثيل عينة الفقرات للأغراض السلوكية، وتتضهن ما يأتي:

الجبوري، مروخ محمد حمد. (2019). إثر استخدام التمثيلات الرياضية في تحصيل تلاميذ الصف الرابع الابتدائي في مادة doi.org/10.36371/port.2018.02.3.12 الرياضيات. Journal Port Science Research ، 
- تعليمات الإجابة: من أجل مساعدة التلاميذ على فهم كيفية الإجابة عن أسئلة الاختبار أعدت الباحثة مجموعة من التعليمات الخاصة بالاختبار، وأعطت فكرة تامة عن الهدف من الاختبار ونوعية الأسئلة وعددها، وزهن الإجابة وتوزيع الدرجات، وعدم اختيار أكثر من إجابة للفقرة الواحدة وعدم ترك أي فقرة من دون إجابة، وتكون الإجابة على ورقة منفصلة يتم فيها كتابة خطوات

$$
\text { الحل من قبل التلميذ. }
$$

- تعليمات التصحيح: أعدت الباحثة مفتاح الإجابات الصحيحة والنهوذجية لفقرات الاختبار، إذ أعطت درجة واحدة للإجابة الصحيحة وصفراً للإجابة غير الصحيحة أو المتروكة للأسئلة الموضوعية، وحددت الدرجة الكلية لها بالمدى (0-28) درجة، أما الفقرات المقالية فحددت اجاباتها في ضوء عدد خطوات الحل الصحيح، فتراوح مداها ما بين (0-15) درجة للفقرة المقالية

$$
\text { الواحدة لتصبح الدرجة الكلية للاختبار (70) درجة. }
$$

تطبيق الاختبار على عينة استطلاعية :طبقت الباحثة الاختبار التحصيلي على عينة استطلاعية اختيرت عشوائياً ، و قد بلغ عدد أفرادها (60) طالبة في مدرسة حاتم الطائي الابتدائية التابعة لمديرية تربية الكرخ الثالثة ، لغرض (حساب الزمن المستغرق للإجابة والتأكد من وضوح الفقرات, طبق الاختبار يوم الاحدالموافق2018/12/23 ، وقد اتضح من التطبيق الاستطلاعي إن جميع الفقرات واضحة

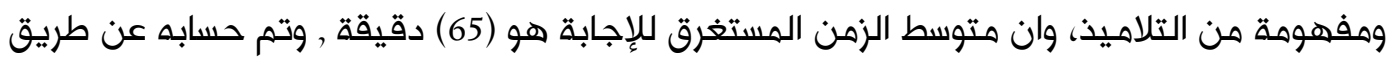
حساب وقت انتهاء أول خمس من التلاميذ ، من الإجابة عن فقرات الاختبار التحصيلي ، واستغرقت (55) دقيقة ، وآخر خمس من التلاميذ إذ استغرقت (75) دقيقة.

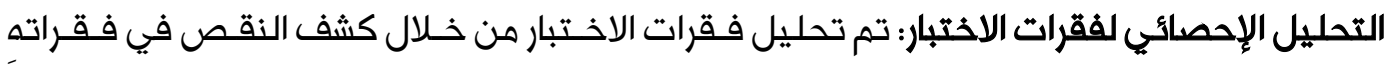
من حـيث القوة والضعف والصياغة لغرض إعـادة صياغتها واسـتبعاد الفـــرات غـير الصالحة منهـا. أ) معامل صعوبة الفقرات: قد حسبت معاملات الصعوبة لكل فقرة من فقرات الاختبار باستخدام معادلة الصعوبة، فوجدت قيمها تتراوح بين (0,73-0,30)، اذ إن الاختبار الجيد هو الذي يتضمن فقرات تتراوح نسبة صعوبتها بين (0,20 و 0,80) (6)، وبهذا تعد فقرات الاختبار مقبولة ومعامل صعوبتها مناسباً من ناحية هذا المؤشر الإحصائي. ب) قوة التمييز: يشير Brown إلى أن الفقرة جيدة إذا كانت قوتها التمييزية (0,20) فما فوق، والفقرة التي تقل قوتها التمييزية عن (0,20) تكون ضعيفة وينصح بحذفها أو تعديلها [34]، وعند حساب القوة التمييزية لكل فقرة من فقرات الاختبار وجد أنها تتراوح بين (0,67-0,33) وبهذا تعد معظم الفقرات تقع ضمن هذا المدى المقبول من (0,20) فما فوق، لذا تعد فقرات الاختبار مقبولة من حيث 
ج) فعالية البدائل الخاطئة للأسئلة الموضوعية: تم ترتيب إجابات التلاهيذ عن فقرات سؤال الاختيار من متعدد كل على انفراد، وقسمت الى مجموعتين عليا ودنيا، وبعد أن تم استخدام معادلة (البدائل الخاطئة)، وجد أن البدائل الخاطئة قد جذبت إليها عدداً من تلاميذ المجموعة الدنيا أكثر من المجموعة العليا، وبهذا تقرر إبقاء البدائل على ما هي عليه.

\section{ثبات الاختبار التحصيلي}

حسبت الباحثة ثبات فقرات الاختبار التحصيلي بمعادلة (كيودر - ريتشاردسون 20) أو (K-R20) إذ بلغ معامل الثبات (0,86) وهـو معامل ثبات جيدا، حيث تعد الاختبارات جيدة إذا كان معامل ثباتها

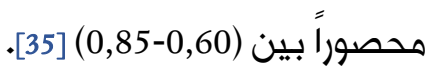

\section{سادساً مرحلة التطبيق:}

1) إجراءات تطبيق التجربة: باشرت الباحثة بتطبيق التجربة على عينة البحث للمجموعتين (التجريبية والضابطة)، ( يوم الاحد الموافق( 2018/10/28), وانتهت في يوم الخميس الموافق( 2019/1/10 )، اذ قامت بتدريس مجموعتي البحث بواقع خمس حصص أسبوعياً لكل مجموعة, درست المجموعة التجريبية الفصول الدراسية الثلاثة (الاحصاء والاحتمالات, الضرب, القسمة) من الكتاب المقرر على وفق استخدام التمثيلات الرياضية , وبحسب الأنشطة والأهداف والاختبارات التي صممتها الباحثة ، و درست الهجموعة الضابطة الفصول ذاتها على وفق الطريقة الاعتيادية.

2) تطبيق الاختبار: بعد الانتهاء من تدريس مفردات المادة الدراسية المحددة، تم إخبار تلاميذ المجموعتين التجريبية والضابطة بأن هناك اختبار سيجري لهم في الفصول الثلاثة، التي تم دراستها خلال الفصل الدراسي بعد أسبوع، وبمساعدة معلمة مادة الرياضيات في المدرسة تم تطبيق الاختبار التحصيلي على المجموعتين في يوم الاربعاء الموافق (2019/1/9) بالنسبة للمجموعتين التجريبية والضابطة.

3) إجراءات التصحيح: بعد الانتهاء من تطبيق الاختبار التحصيلي، قامت الباحثة بتصحيح الاختبار وترتيب الدرجات على شكل جداول، لغرض المعالجة الإحصائية وتفسير النتائج. سابعاً الوسائل الإحصائية استخدمت الباحثة الوسائل الإحصائية الآتية: 1) الاختبار التائي (t-test) لعينتين مستقلتين: أُستخدم لمكافئة مجموعتي البحث في (اختبار المعرفة السابقة في الرياضيات، واختبار الذكاء)، ولمعرفة دلالة الفرق بين متوسطي درجات طالبات مجموعتي البحث في اختبار التحصيل [36].

الجبوري، مروخ محمد حمد. (2019). إثر استخدام التمثيلات الرياضية في تحصيل تلاميذ الصف الرابع الابتدائي في مادة الرياضيات. Journal Port Science Research 


$$
t_{\left(n_{1}+n_{2}-2\right)}=\frac{\bar{x}_{1}-\bar{x}_{2}}{\sqrt{\frac{\left(n_{1}-1\right) s_{1}^{2}+\left(n_{2}-1\right) s_{2}^{2}}{n_{1}+n_{2}-2}\left(\frac{1}{n_{1}}+\frac{1}{n_{2}}\right)}}
$$

الاختبار التائي:

المتوسط الحسابي للمجموعة التجريبية

لمتوسط الحساب للمجموعة الضابطة

الانحراف المعياري للمجموعة التجريبية

الانحراف المعياري للمجموعة الضابطة

عدد طلاب المجموعة التجريبية

عدد طلاب المجموعة الضابطة

2) معامل صعوبة الفقرة: أستخدم لحساب معامل صعوبة الفقرات في اختبار التحصيل [37].

$$
P=\frac{n_{u}+n_{L}}{2 n}=\text { صعوبة الفقرة }
$$

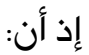

$$
\begin{aligned}
& \boldsymbol{n}_{\boldsymbol{u}}=\text { عدد الإجابات الصحيحة في المجموعة العليا } \\
& \text { عد الإجابات الصحيحة في الهجموعة الدنيا } \\
& \text { ع عدد أفراد إحدى الهجموعتين العليا أو الدنيا }
\end{aligned}
$$

3) معامل تمييز الفقرة: أُستخدم لحساب معامل تمييز الفقرات في اختبار التحصيل [37]

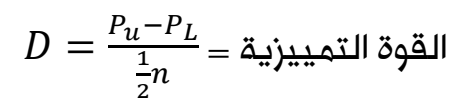

$$
\begin{aligned}
& \text { عدد الإجابات الصحيحة في الهجموعة العليا } \\
& p_{L}=\text { عدد الإجابات الصحيحة في المجموعة الدنيا } \\
& \text { ع عدد أفراد إحدى الهجموعتين العليا أو الدنيا }
\end{aligned}
$$

4) معادلة معامل فعالية البدائل الخاطئة: أُستخدم لإيجاد فعالية البدائل الخاطئة:

$$
D_{A}=\frac{P_{u}-P_{L}}{n}
$$

$$
\text { عد ع الذين اختاروا البديل الخاطئ من المجموعة العليا }
$$

$$
\text { عدد الذين اختاروا البديل الخاطئ من المجموعة الدنيا = عدون }
$$

$$
\text { ع عدد أفراد إحدى المجموعتين }
$$

Al- Jubouri, M. (2019). The Effect of Using Mathematical Representations in the Achievement of Fourth Grade Students in Mathematics. Journal Port Science Research, 2(3), 593-613. doi.org/10.36371/port.2018.02.3.12 
5) معادلة كيودر (يتشاردسون (K-R20) استخدمت لحساب ثبات الاختبار [38]

$$
\begin{aligned}
& R_{20}=\frac{n}{n-1}\left(1-\frac{\sum p \cdot q}{S_{x}^{2}}\right) \\
& \text { عدد الفقرات } \\
& \text { ن =سبة الإجابات الصحيحة عن الفقرة أو السؤال } \\
& q=\text { = نسبة الإجابات الخاطئة عن الفقرة أو السؤال } \\
& \text { التباين الكلي للإجابات = لإجابات }
\end{aligned}
$$

\section{نتائج البحث وتصليلها}

$$
\text { أولاً: عرض النتائج }
$$

التحقق من الفرضية الصفرية التي تنص على انه: لا يوجد فرق ذو دلالة إحصائية عند مستوى دلالة (0,05) بين متوسطي درجات تلاميذ ( المجموعة التجريبية) الذين درسوا وفق استخدام التمثيلات الرياضية ومتوسط درجات تلاميذ ( الهجموعة الضابطة ) الذين درسوا وفق الطريقة الاعتيادية في اختبار التحصيل).تم حساب درجات اختبار التحصيل الكلي لتلاميذ مجموعتي البحث ، وقد بلغ المتوسط الحسابي للدرجات الكلية التي حصل عليها أفراد المجموعة التجريبية $(71,108)$ بانحراف معياري قدره $(11,556)$ وبلغ المتوسط الحسابي للدرجات الكلية التي حصل عليها أفراد المجموعة الضابطة (63,794) بانحراف معياري (11,009) وباستخدام الاختبار التائي (t-Test) لعينتين مستقلتين غير متساويتين بلغت القيمة التائية الهحسوبة (2,704) وهي أكبر من القيمة التائية الجدولية البالغة (2,000) وهي دالة إحصائياً عند مستوى دلالة( 0,05 ) ودرجة حرية ( 69 ) ، ولصالح المجموعة التجريبية , ويدل ذلك إلى وجود فرق دال إحصائياً بين تلاميذ المجموعتين التجريبية والضابطة في الاختبار التحصيلي ، ولصالح المجموعة التجريبية ، مما يدل على أن استخدام التمثيلات الرياضية اثرت بصورة ايجابية في تحصيل التلاميذ في مادة الرياضيات , إذ ينعكس ذلك على النتائج في المتغير أعلاه عند اختبار فرضيته ، وفي ضوء هذه النتيجة ترفض الفرضية الاولى. ثانياً: تفسير النتأُج: أسفرت النتائج عن رفض الفرضية الصفرية وهذا يعني تفوق تلاميذ الهجموعة التجريبية التي تدرس باستخدام التمثيلات الرياضية على تلاميذ المجموعة الضابطة التي تدرس باستخدام الطريقة الاعتيادية للتدريس في التحصيل لتلاهيذ الصف الرابع الابتدائي في مادة 


$$
\text { ويهكن أن تعزى هذه النتيجة إلى الأسباب الآتية: }
$$

1) أن استخدام التمثيلات الرياضية قد ساعدت التلاميذ على القيام بأنشطة من خلال الربط بين الرياضيات وبيئة التلاميذ والى أهمية إعطاء المعلم المتعلم المثيرات المناسبة التي تمكنه من التفكير في المحتوى الدراسي والتفاعل معه للوصول الى فهم المعلومات وتصنيفها وتسميتها

$$
\text { وترتيبها وتنظيمها بشكل منظم. }
$$

2) تعد استخدام التمثيلات الرياضية للتدريس أكثر ملائمة لطبيعة مادة الرياضيات ومن ثم أقرب

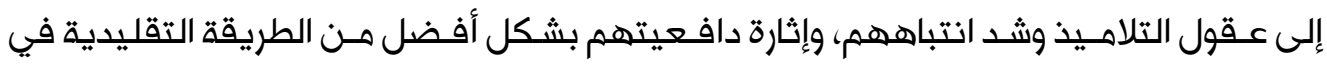
التدريس.

إن التدريس بحسب استخدام التمثيلات الرياضية يجعل التلميذ يربط بين المفاهيم الرئيسة

$$
\text { والفرعية مها يجعل التلمـيذ يمر بإعـادة تعليم مستمر أي يجعل التعلم ذا معنى. }
$$

طبيعة الاجراءات المتبعة في استخدام التمثيلات الرياضية التي تتناول المفهوم الرياضي وتعرضه بصور مختلفة مما يزيد من مرونة التلميذ في التعامل مع الصور المختلفة لذلك المفهوم بالإضافة إلى ذلك فإنها تسمح للتلميذ بتنمية قدراته المعرفية وزيادة فهمه بالإضافة إلى إنها تحوي الصورة الحسية وشبه الحسية والهجردة للأفكار والمفاهيم الرياضية مما يسهل فهمها والانخراط في أنشطتها بالإضافة إلى ميزة الانتقال من تمثيل إلى آخر ينمي قدرات التلميذ بالتعامل مع المفاهيم الرياضية بأي شكل (تمثيل) عرضت بهـ الِّهـ ثالثاً: الاستنتاجات: في ضوء النتائج التي توصل إليه البحث الحالي نستنتج الآتي: 1) استخدام التمثيلات الرياضية لها أثر في رفع مستوى التحصيل في مادة الرياضيات لدى تلاهيذ

$$
\text { الصف الرابع الابتدائي بدرجة أفضل من الطريقة المعتادة في التدريس. }
$$

استخدام التمثيلات الرياضية أثبتت فاعليتها، ضمن الحـدود التي اجـري فيها البحث الحالي وذلك في تحصيل تلاميذ الصف الرابع الابتدائي في مادة الرياضيات مقارنة بالطريقة التقليدية

$$
\text { في التدريس. }
$$

يسـهم استخدام التمثيلات الرياضية في رفـع المستوى التعليمي للتلامـيذ وزيادة فهمهم

$$
\text { للمادة وهـن ثم تثب بيتها في أذهـانهم. }
$$

إن اسـتخدام التمثيلات الرياضية يتطلب من معـلم المادة التخطيط المسبق والدقيق بما يتلاءم

$$
\text { مـع مق تضضيات الـدرس وأهـدافه. }
$$

Al- Jubouri, M. (2019). The Effect of Using Mathematical Representations in the Achievement of Fourth Grade Students in Mathematics. Journal Port Science Research, 2(3), 593-613. doi.org/10.36371/port.2018.02.3.12 
ابتكار أساليب لتقويم تعلم رياضيات المرحلة الابتدائية تراعي من خلالها التمثيلات الرياضية

$$
\text { المتعددة، والتركيز على الأداء الكيفي وليس الكمي فقط. }
$$

2) على المعلمين تدريب تلاميذهم أثناء الحصص على التعبير عن أفكارهم بتمثيلات رياضية

$$
\text { واختبار التمثيل قبل الحكم عليه، ونقله إلى مواقف أخرى. }
$$

3) مناقشة الأمثلة والتمارين في الكتاب المدرسي، وذلك بقراءة التلاميذ لها ثم يطلب منهم التعبير عنها بأسلوبهم شفهياً وإنتاج أكبر عدد ممكن من التمثيلات الرياضية لها في صور مختلفة

$$
\text { (أشكال، جداول، نماذج محسوسة، علاقات رياضية، رموز، مصطلحات، ....). }
$$

4) ضرورة استخدام المعلمين للتمثيلات الرياضية المتعددة سواء كانت ملموسة أو تصويرية، أو رمزية، أو لفظية، أو بصرية، أو داخلية أو خارجية بشكل فعال أثناء تدريس الهحتوى لتحقيق المعرفة العميقة في الرياضيات. 5) ضرورة تبني القائمين على تخطيط المناهج وبنائها للتمثيلات الرياضية عند شرح المفهوم، وإعطاء تمارين مناسبة لتمثيل الفكرة الرياضية بأكثر من شكل 6 ) التركيز بشكل واضح أثناء تدريب المعلمين على وظائف التمثيلات الرياضية في عملية التدريس جنباً إلى جنب مع تدريس المحتوى الرياضي، وعلى تطوير معرفة المعلمين فيما يتعلق

$$
\text { باستخدام التمثيلات الرياضية. }
$$

7) ضرورة الاهتمام بتدريب المعلمين أثناء الخدمة على توظيف التمثيلات الرياضية في تعليم رياضيات المرحلة الابتدائية، فالتلميذة في هذه المرحلة ما زالت في مرحلة العمليات المحسوسة طبقاً لنظرية بياجيه، ولا تفهم الرياضيات إلا من خلال نماذج محسوسة أو أنشطة تعليمية. توصي الباحثة بالاهتمام بالمسائل اللفظية في كل مناهج الرياضيات حتى يظهر المعنى الحقيقي للرياضيات حتى يحبها التلميذات التي تعتبر المسائل اللفظية بمثابة الألغاز التي لا يقدر على حلها سوى النبهاء والموهوبين.

الجبوري، مروخ محمد حمد. (2019). إثر استخدام التمثيلات الرياضية في تحصيل تلاميذ الصف الرابع الابتدائي في مادة

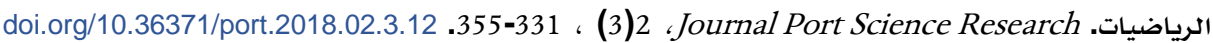




\section{References}

[1] Al-Mashhadani, Abbas Naji. (2011). طرائق ونماذج تعليمية في تدريس الرياضيات >. Oman: Yazouri Scientific House. http://alexir.org/library.php?id=83

[2] Alhila, Mohamed Mahmoud. (2014) > مهارات التدريس الصفي > Oman: Dar march publishing, distribution and printing. https://jamalon.com/en/1023754.html

[3] Ali, Mohamed El Sayed. (2018). > اتجاهات وتطبيقات حديثة في المناهج وطرق التدريس . Oman: Dar march publishing, distribution and printing. https: / / www.neelwafurat.com/itempage.aspx?id=lbb199914-171577\&search=books

[4] El Sherbiny, Fawzy. Eltnawy, Effat Mustafa. (2011) > اتجاهات وتطبيقات حديثة في الهناهج تطوير >. Oman: Dar march publishing, distribution and printing. https: / / www.neelwafurat.com/itempage.aspx?id=lbb194231-1639198search=books

[5] Alhila, Mohamed Mahmoud. (2011). طرائق التدريس واستراتيجياته > Al Ain: UNIVERSITY BOOK HOUSE. http: / / altibrah.ae/book/2188

[6] Al Kubaisi Abdel Wahed. (2015). > القياس والتقويم - تجديدات ومناقشات > Dar Jarir Publishing \& Distribution. https: / / www.neelwafurat.com/itempage.aspx?id=lbb169774-132639\&search=books

[7] Al-Azzawi, Hani Kamal. (2003). The teaching competencies for the chemistry teachers and their relation with the student trends towards the chemistry. (University Thesis), University Baghdad University, College of Education for Pure Sciences - Ibn al-Haytham. http:/ / search.mandumah.com/Record/558965

[8] Ibrahim, Mohammed Khader. (2001). مقدرة طلبة الصفين السابع والثامن الأساسي على التمثيل الجبري : والهندسي للمسألة الرياضية اللفظية > . (Master Thesis) Yarmouk University, Jordan.

[9] Bedair, Kariman Mohammed. (2012). التعلم النشط> > . Your Guide and Directory Dar Al - Masira Publishing, Distribution and Printing, Amman.

[10] Ibrahim, Bassam Abdullah Taha. (2009). التعلم المبني على المشكلات الحياتية> > Your Guide and Directory Dar Al - Masira Publishing, Distribution and Printing, Amman.

[11] Herrnstein, R. J., Nickerson, R. S., de Sanchez, M., \& Swets, J. A. (1986). Teaching Thinking Skills. American Psychologist, 41(11), 1279-1289. https: / / doi.org/10.1037/0003-066X.41.11.1279

[12] Ghabari, Thaer Ahmed Fadil. Abu Shaira, Khaled Mohammed Abdel Fattah. (2015). أساسيات في >. Amman, Jordan: Dar Al-Huron Scientific Publishing and Distribution: Arab Society Library for Publishing and Distribution, https://library.nauss.edu.sa/cgi-bin/koha/opacdetail.pl?biblionumber $=25299$

[13] Al-Sawai, Othman. (2010) مهارات التمثيل الرياضي وإجراء العمليات الحسابية لدى طلاب الصف السادس (20) . الابتدائي ، مجلة العلوم التربوية والنفسية. College of Education, University of Bahrain. 11(3).

Al- Jubouri, M. (2019). The Effect of Using Mathematical Representations in the Achievement of Fourth Grade Students in Mathematics. Journal Port Science Research, 2(3), 593-613. doi.org/10.36371/port.2018.02.3.12 
[14] Hwang, W. Y., Chen, N. S., Dung, J. J., \& Yang, Y. L. (2007, April). Multiple representation skills and creativity effects on mathematical problem solving using a multimedia whiteboard system. Educational Technology and Society.

[15] Bhot, Abdul Jawad. Mohamed, Abdelkader (2005) تأثير استخدام مدخل التمثيلات الرياضية على بعض > مهارات التواصل الرياضي لدى تلاهيذ الصف السادس الابتدائي . Fifth Scientific Conference (Global and Educational Changes and Mathematics Education), Egyptian Association for Mathematics Education, Egypt.

[16] Badawi, Ramadan Saad. (2003). إستراتيجيات في تعليم وتقويم تعلم الرياضيات > Your Guide And Directory Dar Al - Fikr for Printing, Publishing and Distribution, Amman.

[17] Schunk, D. H. (2012). Learning theories: An educational perspective. (M. Harlan, Ed.), Reading (Vol. 5, p. 578). Pearson. Retrieved from http://www.amazon.com/Learning-TheoriesEducational-Perspective-6th/dp/0137071957

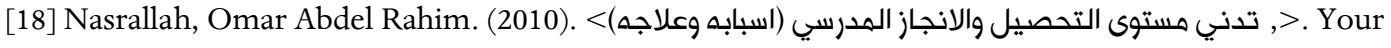
Guide and Directory Dar Wael for Publishing and Distribution, Amman.

[19] Bengio, Y., Courville, A., \& Vincent, P. (2013). Representation learning: A review and new perspectives. IEEE Transactions on Pattern Analysis and Machine Intelligence, 35(8), 1798-1828. https:// doi.org/10.1109/TPAMI.2013.50

[20] Pape, S. J., \& Tchoshanov, M. A. (2001). The role of representation(s) in developing mathematical understanding. Theory into Practice, 4O(2), 118-127. https://doi.org/10.1207/s15430421tip4002_6

[21] Al-Bilasi, Riad Ibrahim; Barham, Areej Essam. (2010). The effect of suing multiple mathematical representations on the attainment of mathematical concepts among basic eight grade students and their ability to solve verbal problems. Dirasat: Educational Sciences. 2010, Vol. 37 Issue 1, p1-13. 13p. http://search.shamaa.org/FullRecord?ID=79924

[22] Chen, C. J. (2016). Mathematical Representations. In Elements of Human Voice (pp. 97-98). WORLD SCIENTIFIC. https:// doi.org/10.1142/9789814733908_others02

[23] Badawi, Ramadan Saad. (2007) تدريس الرياضيات الفعال من رياض الاطفال حتى الصف السادس الابتدائي>. Dar Al Fikr Publishers and Distributors. https://www.daralfiker.com/node/6606

[24] Geary, D. C. (2007). Development of Mathematical Understanding. In Handbook of Child Psychology. John Wiley \& Sons, Inc. https:// doi.org/10.1002/9780470147658.chpsy0218

[25] Suh, J. M., \& Moyer-Packenham, P. S. (2007). THE APPLICATION OF DUAL CODING THEORY IN MULTI-REPRESENTATIONAL VIRTUAL MATHEMATICS ENVIRONMENTS. Unknown, 4(1994), 209-216.

[26] Abu Al-Ajeen, Ashraf Hassan Hussein. (2011). Assessment of Palestinian mathematics curricula in light of some processes standards of National Council if Teachers of Mathematics (NCTM) [Thesis / Dissertation]. Curriculum and Instruction. Al-Azhar University - Gaza). Faculty of Education. http://search.shamaa.org/FullRecord?ID =75744

الجبوري، مروخ محمد حمد. (2019). إثر استخدام التمثيلات الرياضية في تحصيل تلاميذ الصف الرابع الابتدائي في مادة الرياضيات. Journal Port Science Research 
[27] Sun, Y., Kulm, G., \& Capraro, M.M. (2009). Middle Grade Teachers' Use of Textbooks and Their Classroom Instruction. https://www.semanticscholar.org/paper/Middle-Grade-Teachers'-Use-ofTextbooks-and-Their-Sun-Kulm/b912aeba4cf908bda3083ef06396e6749abdf5b4

[28] Al-Zaher, Zakaria Ahmed. (2002). مبادئ القياس والتقويم في التربية / تأليف زكريا محمد الظاهر.جاكلين 年 > Amman, Jordan, Publisher International Scientific House and Culture House Publishing. http://213.6.8.28:310/records/1/3594.aspx

[29] Issawi, Abdul Rahman Mohammed. (2000). الطريق الى النبوغ العلمي> > Beirut, [Lebanon]: University salary house. https:/ / libserver.bethlehem.edu/webopac/records/1/63368.aspx

[30] Saidi, Khalifa bin Abdullah. (2010) فاعلية استخدام التمثيلات الرياضية المتعددة في تنمية مهارات حل ) المشكلات الرياضية لدى طلاب الصف الثامن من التعليم الأساسي . (Master Thesis), Faculty of Educational Sciences, Jordan.

[31] Novotna, J. (2006). Proceedings of the Conference of the International Group for the Psychology of Mathematics Education (30th, Prague, Czech Republic, July 16-21, 2006). Volume 1. In Proceedings of the Conference of the International Group for the Psychology of Mathematics Education (30th, Prague, Czech Republic, July 16-21, 2006). Volume 1(p. 542). Retrieved from http: / / eric.ed.gov/ ERICWebPortal/ detail?accno=ED496931

[32] Küchemann, D., Hodgen, J., \& Brown, M.L. (2011). Models and representations for the learning of multiplicative reasoning: Making sense using the Double Number Line. https: / / www.semanticscholar.org/paper/Models-and-representations-for-the-learning-of-theK\%C3\%BCchemann-Hodgen/7343542b83e8231aa3e614bf950dbc3ff9221606

[33] Jassem, Amir Abdul Majeed and others. (2018). سلسلة كتب الرياضيات للمرحلة الابتدائية> > Directorate General of Curricula, Baghdad.

[34] Golding, C., \& Adam, L. (2016). Evaluate to improve: useful approaches to student evaluation. Assessment and Evaluation in Higher Education, 41(1), 1-14. https: / / doi.org/10.1080/02602938.2014.976810

[35] Stiggins, R. J., Griswold, M. M., \& Wikelund, K. R. (1989). Measuring Thinking Skills Through Classroom Assessment. Journal of Educational Measurement, 26(3), 233-246. https: / / doi.org/10.1111/j.1745-3984.1989.tb00330.x

[36] Gudgeon, A. C., \& Howell, D. C. (1994). Statistical Methods for Psychology. The Statistician, 43(1), 211. https: / / doi.org/ $10.2307 / 2348956$

[37] Odeh, Ahmad Suleiman. (1999). القياس والتقويم في العملية التدريسية> > Dar Al-Amal for Publishing and Distribution, Amman.

[38] Melhem, Sami Mohamed. (2002). سيكولوجية التعلم و التعليم "الأسس النظرية و التطبيقية> Dar Al Massira, Amman.

Al- Jubouri, M. (2019). The Effect of Using Mathematical Representations in the Achievement of Fourth Grade Students in Mathematics. Journal Port Science Research, 2(3), 593-613. doi.org/10.36371/port.2018.02.3.12 